\title{
Peptídeo C e mortalidade cardiovascular: revisão sistemática e metanálise
}

\author{
Romildo Luiz Monteiro Andrade, ${ }^{1}$ Gabriela Callo e Bernardo Lessa Horta ${ }^{2}$
}

Como citar Andrade RLM, Callo G, Horta BL. Peptídeo C e mortalidade cardiovascular: revisão sistemática e metanálise. Rev Panam Salud Publica. 2019;43:e23. https://doi.org/10.26633/RPSP.2019.23

RESUMO

Objetivo. Analisar as evidências de associação entre peptídeo C e mortalidade cardiovascular e geral disponíveis na literatura.

Métodos. Foi realizada uma revisão sistemática das bases de dados MEDLINE e EMBASE. Foram incluídos artigos publicados em inglês, português ou espanhol relatando estudos observacionais que investigaram a associação entre o peptídeo C e a mortalidade cardiovascular e geral. Buscou-se ainda avaliar a associação entre peptídeo $\mathrm{C}$ e fatores de risco cardiometabólicos, fatores hemodinâmicos e medidas antropométricas. $\mathrm{A}$ qualidade metodológica dos estudos foi avaliada de acordo com os critérios da escala Newcastle-Ottawa.

Resultados. Foram identificados 107 estudos relacionados ao tema. Ao final do processo de triagem, foram incluídos 18 artigos que apresentavam dados sobre a associação entre peptídeo C e risco cardiovascular. Cinco estudos forneceram dados sobre a relação entre peptídeo C e mortalidade cardiovascular e geral. O peptídeo C esteve associado positivamente ao IMC em chineses, e inversamente ao HDL colesterol em amostras populacionais na Ásia, Oriente Médio e Estados Unidos. Todavia, não foi possível realizar metanálise para os componentes de risco cardiovascular. Por outro lado, o peptídeo C esteve associado com a mortalidade cardiovascular ( $R R=1,62$; IC95\%: 0,99 a 2,66) e geral ( $R R=1,39 ;$ IC95\%: 1,04 a 1,84).

Conclusão. Na revisão sistemática e metanálise realizadas, os níveis séricos de peptídeo C estiveram associados positivamente com a mortalidade geral em todos os indivíduos e com a mortalidade cardiovascular em pessoas sem comorbidades. Com base nesses resultados, é possível recomendar o emprego do peptídeo C na prática clínica como proxy da condição de resistência à insulina associada a mortalidade cardiovascular.

Palavras-chave Peptídeo C; mortalidade; doenças cardiovasculares; metanálise.

Em 1967, Steiner et al. descreveram a síntese da insulina humana, identificando a molécula da pró-insulina como seu precursor (1). Por sua vez, o peptídeo de conexão da insulina (peptídeo C) foi apontado como molécula de ligação entre as cadeias A e B da insulina. Durante décadas, o peptídeo C foi considerado como um subproduto organicamente inerte da clivagem da pró-insulina, sem propriedades fisiológicas (2-4). Posteriormente, estudos em animais e humanos (5-7) observaram que o peptídeo $C$ pouco influenciava o controle glicêmico, mas evitava complicações microangiopáticas como nefropatia diabética em portadores de diabetes tipo 1 (DM1) (8-10). Um ensaio clínico apontou benefícios como diminuição da incidência de complicações microvasculares e da homeostase

\footnotetext{
1 Universidade Federal do Espírito Santo, Hospital Universitário Cassiano
} Antonio de Moraes, Vitória (ES), Brasil. $\square$ rlandrade@hotmail.com metabólica em indivíduos com níveis séricos e secreção preservada do peptídeo C (11). Por outro lado, Cai et al. relataram que menores níveis de peptídeo C pós-prandial e idade de diagnóstico estariam associados a maior prevalência de retinopatia diabética (12).

Recentemente, outros estudos identificaram que o peptídeo C estaria associado à atividade inflamatória vascular e ao processo aterosclerótico (13). Sua atuação permitiria o aumento da permeabilidade vascular para monócitos, estimulando a diferenciação em macrófagos, favorecendo a fagocitose de moléculas como a lipoproteína de baixa densidade (LDL), contribuindo para a diferenciação em células espumosas, que se constituem no substrato celular clássico das lesões ateroscleróticas. Ainda 
em fases subsequentes do processo aterogênico, o peptídeo $\mathrm{C}$ induziria a proliferação das células musculares na cascata pró-aterogênica, liberando citocinas, metaloproteínas e moléculas oxidativas, além de fatores estimulantes da coagulação, como o ativador do plasminogênio tecidual (tPA) $(14,15)$.

O peptídeo C é secretado em quantidades equimolares da insulina e sua dosagem sérica tem sido empregada na prática clínica, dentre outras finalidades, para estimar a capacidade de produção do pâncreas endócrino (16). Os níveis de peptídeo C estão associados ao índice de massa corpórea (IMC), ao tempo de evolução do diabetes tipo 2 (DM2) e à condição de resistência à insulina (RI). Por sua vez, a RI favorece a hiperinsulinemia, que cursa com elevação sérica do peptídeo C, contribuindo para disfunção endotelial, desencadeando processos inflamatórios endovasculares e elevando o risco cardiovascular (17). Von Eckardstein et al. estudaram o papel do peptídeo C como fator de risco cardiovascular em homens coronariopatas não diabéticos, observando associação com doença aterosclerótica coronariana (DAC) (18). Cabrera et al. observaram, na população geral, que o peptídeo C aumentava a incidência de infarto do miocárdio e de DAC (19).

Considerando o crescente interesse na identificação de novas vias cardiometabólicas que possam contribuir para a compreensão da relação entre os fatores de risco clássicos e novos alvos para a prevenção das doenças cardiovasculares, o objetivo da presente revisão foi avaliar as evidências referentes à associação entre o peptídeo $\mathrm{C}$ e a mortalidade cardiovascular.

\section{MATERIAIS E MÉTODOS}

Foi realizada uma revisão sistemática das bases de dados MEDLINE (https://www.nlm.nih.gov/bsd/medline.html) e EMBASE (https://www.elsevier.com/solutions/embasebiomedical-research\#GetStarted). Além de artigos publicados em periódicos científicos, a revisão buscou livros, jornais e páginas da rede sobre o tema. Também foram pesquisados os sites da World Health Organization (WHO) - tópicos Health, Cardiovascular diseases, Technical information, Global Hearts Initiative e Technical package for cardiovascular disease (20) -, da American Heart Association e das sociedades europeia e brasileira de cardiologia. As referências bibliográficas dos artigos selecionados também foram examinadas.

Foram incluídos estudos observacionais que investigaram a associação entre o peptídeo $\mathrm{C}$ e a mortalidade cardiovascular e geral e estudos sobre a ocorrência de desfechos cardiovasculares, incluindo eventos cardiovasculares como síndromes coronarianas agudas, compreendendo as formas de angina do peito e infarto do miocárdio. Foram excluídos ensaios clínicos não controlados que avaliaram indivíduos submetidos a terapias dietéticas e acompanhados antes e depois, assim como estudos que avaliaram subgrupos específicos de patologias não vinculadas ao recorte cardiovascular ou grupos de indivíduos como renais crônicos, portadores de artrite reumatoide, hipotireoidismo ou obesidade associada a condição de RI. Por se tratarem de estudos observacionais, a presente revisão sistemática seguiu as recomendações propostas pela diretriz MOOSE (21) para seleção, análise e elaboração dos resultados.

\section{Estratégia de busca}

As buscas foram realizadas de forma independente por dois pesquisadores, sem restrições quanto ao ano de início (busca realizada desde a origem da MEDLINE, em 1900, e a partir de 1988 na EMBASE). Para ambas as bases, a busca foi encerrada em julho de 2017. Foram incluídos artigos publicados em inglês, português ou espanhol. A estratégia de busca utilizada em ambos os sítios de pesquisa empregou Medical Subject Headings $(\mathrm{MeSH})$ referentes a ("cardiovascular system") OR ("cardiovascular" e "system") AND ("mortality") AND ("C-Peptide" OR "c peptide"). Na seleção inicial, aplicaram-se filtros adicionais para restringir a busca a estudos envolvendo seres humanos. Os artigos em duplicidade foram excluídos, sendo selecionados estudos conduzidos sem restrições geográficas ou limitações demográficas de raça ou cor. Eventuais discordâncias entre os pesquisadores foram solucionadas por um terceiro avaliador (BLH). Posteriormente, os estudos foram organizados cronologicamente segundo data de publicação, autoria e país de estudo, desenho metodológico, população investigada, forma de emprego do peptídeo C, associação estudada e resultados encontrados (tabela 1).

\section{Exposição e desfecho}

O peptídeo C como variável de exposição foi avaliado em quartil, tercil ou na forma de variável contínua. Os desfechos principais foram mortalidade cardiovascular e geral. Também foram avaliados estudos da associação entre peptídeo $\mathrm{C}$ e fatores metabólicos para risco cardiovascular, como triglicerídeos, glicemia, lipoproteína de alta densidade (HDL), proteína C reativa, leptina, índice aterogênico, triglicerídeos/HDL, ácido úrico, fatores hemodinâmicos, como pressão arterial sistólica e diastólica, e medidas antropométricas, como circunferência da cintura e IMC.

A qualidade metodológica dos estudos foi avaliada de acordo com os critérios da Newcastle-Ottawa Scale (NOS) (22). Foi avaliada a representatividade amostral do grupo dos expostos (descrição do grupo do qual foram extraídos os indivíduos expostos, seu acompanhamento e forma de seleção) e do grupo dos não expostos (descrição do grupo do qual foram extraídos os indivíduos não expostos, seu acompanhamento e forma de seleção).

Para a representatividade amostral, consideraram-se como atendidos os critérios (atendido: sim/não) caso a seleção dos participantes tivesse respeitado os princípios da equiprobabilidade amostral mediante descrição dos seguintes itens:

- critérios de exposição: determinação do peptídeo C a partir da realização de exames ou autorrelato;

- demonstração de que o resultado de interesse não estava presente no início do estudo;

- estabelecimento de medidas de controle para confundidores, como restrição, pareamento ou randomização;

- avaliação cega do desfecho ou mediante referência do participante (autorrelato);

- tempo de seguimento para a ocorrência do desfecho investigado de pelo menos 5 anos;

- descrição da taxa de acompanhamento e das perdas.

\section{Análise estatística}

Inicialmente, os estudos foram combinados usando modelo fixo. A heterogeneidade entre os estudos foi avaliada usando o teste $\mathrm{Q}$ de Cochran e a medida do $\mathrm{I}^{2}$ de Higgins e Thompson. 
TABELA 1. Artigos selecionados através da revisão sistemática sobre associação entre peptídeo C e mortalidade cardiovasculara

\begin{tabular}{|c|c|c|c|c|c|c|}
\hline Autor, país, ano & $\begin{array}{l}\text { Desenho, população } \\
\text { investigada }\end{array}$ & $\begin{array}{l}\text { Emprego do } \\
\text { peptídeo C }\end{array}$ & Associação estudada & $\begin{array}{l}\text { Medida de } \\
\text { associação }\end{array}$ & Resultados $^{b}$ & $\begin{array}{l}\text { Escore de } \\
\text { qualidade }\end{array}$ \\
\hline $\begin{array}{l}\text { Pontiroli et al. (27) } \\
\text { Itália } 1997\end{array}$ & $\begin{array}{l}\text { Coorte } \\
93 \text { diabéticos } \\
\text { tipo } 2\end{array}$ & $\begin{array}{l}\text { Exposição } \\
\text { (contínua) }\end{array}$ & DCV & RO & R0 1,47; IC95\%: 1,16 a 1,87 & 3 \\
\hline $\begin{array}{l}\text { Chen et al. (23) } \\
\text { China } 1999\end{array}$ & $\begin{array}{l}\text { Transversal } \\
3247 \text { participantes }\end{array}$ & $\begin{array}{l}\text { Desfecho } \\
\text { (contínuo) }\end{array}$ & $\begin{array}{l}\text { IMC, relação cintura- } \\
\text { quadril, pressão arterial } \\
\text { média, colesterol, TGC, } \\
\text { HDL }\end{array}$ & $\begin{array}{l}\text { Coeficiente de } \\
\text { regressão }\end{array}$ & $\begin{array}{l}\text { IMC: } \beta 0,008 ; P<0,001 \\
\text { Relação cintura-quadril: } \beta 0,037 ; P=0,544 \\
\text { Pressão arterial média: } \beta 0,0008 ; P=0,003 \\
\text { Colesterol: } \beta-0,007 ; P=0,024 \\
\text { TGC: } \beta 0,047 ; P<0,001 \\
\text { HDL: } \beta-0,046 ; P<0,001\end{array}$ & 5 \\
\hline $\begin{array}{l}\text { Haban et al. (15) } \\
\text { Polônia } 2002\end{array}$ & $\begin{array}{l}\text { Transversal } \\
29 \text { diabéticos tipo } 2\end{array}$ & $\begin{array}{l}\text { Exposição } \\
\text { (categórica) } \\
<0,56 \mathrm{nmol} / \mathrm{L} \text { e } \\
\geq 0,56 \mathrm{nmol} / \mathrm{L} \text { ) }\end{array}$ & $\begin{array}{l}\text { Fatores de risco } \\
\text { cardiovascular: } \\
\text { leptina, IMC, TGC, HDL, } \\
\text { índice aterogênico, razão } \\
\text { TGC/HDL, ácido úrico, } \\
\text { glicemia }\end{array}$ & $\begin{array}{l}\text { Coeficiente de } \\
\text { correlação }\end{array}$ & $\begin{array}{l}\text { Leptina: } r=0,492 ; P=0,007 \\
\text { IMC: } r=0,519 ; P=0,004 \\
\text { Triglicerídeos: } r=0,474 ; P=0,009 \\
\text { HDL: } r=-0,567 ; P=0,001 \\
\text { Índice aterogênico: } r=0,599 ; P<0,001 \\
\text { TGC/HDL: } r=0,587 ; P<0,001 \\
\text { Ácido úrico: } r=0,374 ; P=0,046 \\
\text { Glicemia: } r=0,406 ; P=0,029\end{array}$ & 2 \\
\hline $\begin{array}{l}\text { Chan et al. (24) } \\
\text { China } 2004\end{array}$ & $\begin{array}{l}\text { Transversal } \\
521 \text { diabéticos tipo } 2\end{array}$ & $\begin{array}{l}\text { Desfecho } \\
\text { (contínuo) }\end{array}$ & $\begin{array}{l}\text { IMC } \\
\text { baixo: }<18,5 \mathrm{~kg} / \mathrm{m}^{2} \\
\text { normal: } 18,5 \mathrm{a} 23 \mathrm{~kg} / \mathrm{m}^{2} \\
\text { excesso: }>23 \mathrm{~kg} / \mathrm{m}^{2}\end{array}$ & Média geométrica & $\begin{array}{l}\text { IMC x Pep-C: } r=0,250 ; P<0,001 \\
\text { IMC - grupos }(P<0,001) \text { : } \\
\text { baixo IMC: } \mu \text { g 0,63; DP anti-log 3,08 } \\
\text { IMC normal: } \mu \text { g 1,16; DP anti-log } 2,79 \\
\text { excesso: } \mu \mathrm{g} 1,66 ; \text { DP anti-log 2,75 }\end{array}$ & 5 \\
\hline $\begin{array}{l}\text { Hirai et al. (29) } \\
\text { Estados Unidos } 2008\end{array}$ & $\begin{array}{l}\text { Coorte } \\
1007 \text { diabéticos }\end{array}$ & $\begin{array}{l}\text { Exposição } \\
\text { (contínua) }\end{array}$ & $\begin{array}{l}\text { Mortalidade geral, por } \\
\text { DCl e AVE }\end{array}$ & HR & $\begin{array}{l}\text { Mortalidade geral: } \\
\text { Homem: HR 1,19; IC95\%: 0,99 a 1,41 } \\
\text { Mulher: HR 1,18; IC95\%: 1,03 a 1,36 } \\
\text { Mortalidade por DCI: } \\
\text { Homem: HR 1,43; IC95\%: } 1,13 \text { a 1,83 } \\
\text { Mulher: HR 1,09; IC95\%: 0,88 a 1,36 } \\
\text { Mortalidade por AVE: } \\
\text { Homem: HR 1,65; IC95\%: } 1,07 \text { a } 2,53 \\
\text { Mulher: HR 0,99; IC95\%: 0,71 a 1,39 }\end{array}$ & 8 \\
\hline $\begin{array}{l}\text { Bo et al. (28) } \\
\text { Itália } 2012\end{array}$ & $\begin{array}{l}\text { Coorte } \\
931 \text { diabéticos tipo } 2\end{array}$ & $\begin{array}{l}\text { Exposição (tercil) } \\
\text { Ref: } 1^{\circ} \text { tercil }\end{array}$ & Mortalidade geral e DCV & $H R$ & $\begin{array}{l}\text { Mortalidade: sem associação } \\
\text { Complicações crônicas: } \\
\text { - retinopatia: } \\
2^{\circ} \text { tercil: HR 0,51; IC95\%: 0,38 a 0,68 } \\
\text { 30 tercil: HR 0,33; IC95\%: 0,23 a 0,47 } \\
\text { - nefropatia: } \\
2^{0} \text { tercil: HR 0,40; IC95\%: 0,29 a 0,54 } \\
3^{0} \text { tercil: HR 0,27; IC95\%: 0,18 a 0,38 } \\
\text { - neuropatia: } \\
2^{\circ} \text { tercil: HR 0,72; IC95\%: 0,50 a 1,04 } \\
3^{0} \text { tercil: HR 0,39; IC 95\%: 0,25 a 0,61 } \\
\text { - outras complicações: } \\
2^{\circ} \text { tercil: HR 0,72; IC95\%: 0,60 a 0,88 } \\
3^{0} \text { tercil: HR 0,52; IC95\%: 0,42 a 0,65 } \\
\text { Sem associação com DCV }\end{array}$ & 6 \\
\hline $\begin{array}{l}\text { Patel et al. (30) } \\
\text { Estados Unidos } 2012\end{array}$ & $\begin{array}{l}\text { Coorte } \\
5153 \text { participantes }\end{array}$ & $\begin{array}{l}\text { Exposição } \\
\text { (quartil) } \\
\text { Ref: } 1^{\circ} \text { quartil }\end{array}$ & $\begin{array}{l}\text { Mortalidade geral, } \\
\text { mortalidade } \\
\text { cardiovascular }\end{array}$ & $H R$ & 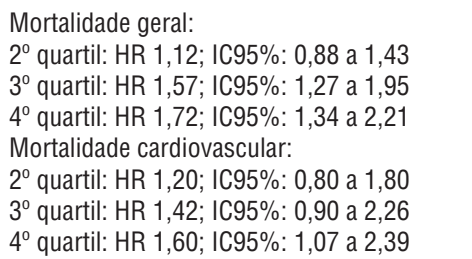 & 8 \\
\hline
\end{tabular}


TABELA 1. (Continued)

\begin{tabular}{|c|c|c|c|}
\hline Autor, país, ano & $\begin{array}{c}\text { Desenho, população } \\
\text { investigada }\end{array}$ & $\begin{array}{l}\text { Emprego do } \\
\text { peptídeo C }\end{array}$ & Associação estudada \\
\hline $\begin{array}{l}\text { Min e Min (33) } \\
\text { Estados Unidos } 2013\end{array}$ & $\begin{array}{l}\text { Coorte } \\
5902 \text { participantes }\end{array}$ & $\begin{array}{l}\text { Exposição (quartil) } \\
\text { Ref: } 1^{\circ} \text { quartil }\end{array}$ & $\begin{array}{l}\text { Mortalidade geral, } \\
\text { cardiovascular e doença } \\
\text { arterial coronariana }\end{array}$ \\
\hline
\end{tabular}

Medida de
associação
HR

\begin{tabular}{|c|c|c|c|c|c|c|}
\hline & & & & & $4^{\circ}$ quartil: HR 2,73 ; IC95\%: 1,55 a 4,82 & \\
\hline $\begin{array}{l}\text { Li et al. (37) } \\
\text { EUA } 2014\end{array}$ & $\begin{array}{l}\text { Transversal } \\
7030 \text { participantes }\end{array}$ & $\begin{array}{l}\text { Exposição } \\
\text { (contínua) }\end{array}$ & AVE & Ro & R0 3,71; IC95\%: 1,78 a 7,75 & 8 \\
\hline $\begin{array}{l}\text { Pikkemaat et al. (32) } \\
\text { Suécia } 2015\end{array}$ & $\begin{array}{l}\text { Coorte } 399 \\
\text { diabéticos tipo } 2\end{array}$ & $\begin{array}{l}\text { Exposição (quartil) } \\
\text { Ref: } 1^{\circ} \text { quartil }\end{array}$ & Mortalidade geral e DCV & $\mathrm{HR}$ & $\begin{array}{l}\text { Mortalidade geral: } \\
2^{\circ} \text { quartil: HR 0,61; IC95\%: 0,05 a 7,79 } \\
3^{0} \text { quartil: HR 1,06; IC95\%: 0,13 a 8,65 } \\
4^{0} \text { quartil: HR 2,67; IC95\%: 0,40 a 17,75 } \\
\text { DCV: sem associação }\end{array}$ & 8 \\
\hline $\begin{array}{l}\text { Cardellini et al. (34) } \\
\text { Itália } 2017\end{array}$ & $\begin{array}{l}\text { Coorte } 431 \\
\text { portadores de DAC }\end{array}$ & $\begin{array}{l}\text { Exposição } \\
\text { (contínua) }\end{array}$ & $\begin{array}{l}\text { Mortalidade geral, } \\
\text { mortalidade } \\
\text { cardiovascular }\end{array}$ & $\mathrm{HR}$ & $\begin{array}{l}\text { Mortalidade geral: } \\
\text { HR 1,38; IC95\%: } 1,06 \text { a } 1.80 \\
\text { Mortalidade cardiovascular: } \\
\text { HR 1,09; IC95\%: 0,90 a } 1,32\end{array}$ & 8 \\
\hline
\end{tabular}

a AVE: acidente vascular encefálico; CC: circunferência da cintura; DAC: doença aterosclerótica coronariana; DCI: doença cardíaca isquêmica; DCV: doença cerebrovascular; HDL: lipoproteínas de alta densidade; HR: hazard ratio;

IMC: índice de massa corporal; PAD: pressão arterial diastólica; RO: razão de odds; RR: risco relativo; TGC: triglicerídeos.
${ }^{b} \mu$ : Média; $\beta$ : coeficiente de regressão; $r$ : coeficiente de correlação; $\mu$ g: média geométrica.

Como a heterogeneidade foi elevada, combinamos os estudos usando modelo randômico. Todas as análises foram conduzidas utilizando o software Stata, versão 13.0.

\section{RESULTADOS}

A partir da pesquisa eletrônica, identificamos 3461 estudos, 1414 oriundos da EMBASE e 2047 provenientes do MEDLINE. A figura 1 apresenta o processo de seleção dos estudos após aplicação dos critérios de elegibilidade. Foram selecionados 107 estudos para leitura de títulos e resumos. Desses, 32 estudos abordavam o peptídeo $C$ como condição de exposição: seis foram excluídos por serem estudos de revisão, um por investigar subgrupos com comorbidades específicas de hipotireoidismo, dois por investigarem a obesidade em crianças submetidas a intervenção dietética e cinco por investigarem a associação do peptídeo $\mathrm{C}$ a receptores de membrana, cafeína e outras condições patológicas como câncer de cólon e pulmão. Sendo assim, 18 estudos foram selecionados para a presente revisão $(12,15,19$, 23-37).

A síntese das principais características dos estudos e seus achados estão representados na tabela 1 . Um estudo avaliou o efeito protetor da função residual das células $\beta$ através do peptídeo $C$ no desenvolvimento de complicações microvasculares em indivíduos com DM1. Dois estudos investigaram a associação do peptídeo C com o IMC, um com a gordura corporal e três com o HDL; além desses, quatro estudos examinaram a associação entre o peptídeo $C$ e a pressão arterial sistólica e cinco entre o peptídeo $\mathrm{C}$ e a pressão arterial diastólica. Oito estudos investigaram o peptídeo $\mathrm{C}$ e seus efeitos sobre a mortalidade 
FIGURA 1. Fluxograma da seleção de artigos, revisão sistemática sobre associação entre peptídeo C e mortalidade cardiovascular

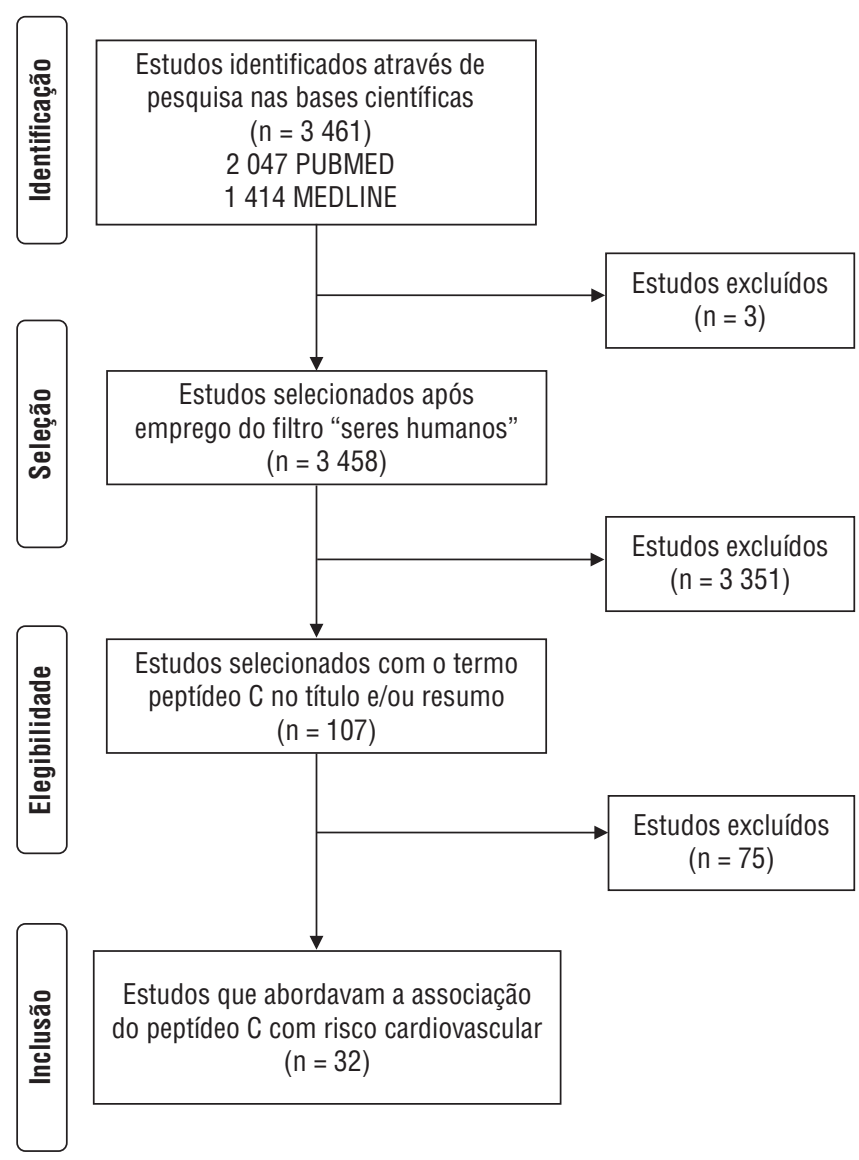

geral, e cinco investigaram os efeitos sobre a mortalidade cardiovascular.

\section{Peptídeo C e condições de risco cardiovascular}

Chen et al. (23) demonstraram, em chineses com tolerância normal à glicose, que o peptídeo $\mathrm{C}$ em jejum está associado ao IMC $(P<0,001)$. A associação foi mais forte entre as mulheres. Chan et al. (24) acompanharam 521 diabéticos em Hong Kong e também identificaram associação linear entre o peptídeo $C$ e o $\operatorname{IMC}(P<0,001)$, mesmo após ajuste para condição de resistência à insulina. Todavia, a disponibilidade dos dados em diferentes categorias de peptídeo C não permitiu agregar os estudos em uma metanálise.

No que se refere ao HDL colesterol, Abdullah et al. (25) encontraram associação inversa com o peptídeo $C(\beta-9,46 ; P=$ $0,01)$. Chen et al. (23) também observaram associação inversa entre o peptídeo C e o HDL ( $\beta-0.04 ; P<0,001)$, assim como Li et al. (26) em 13185 participantes do National Health and Nutrition Examination Survey III (NHANES III) $(P=0,001)$. No estudo de Li et al. (26), os homens tiveram chance de 3,07 (IC95\%: 2,55 a 3,70), e as mulheres de 3,67 (IC95\%: 3,08 a 4,37) para os níveis mais altos do peptídeo C ( $4^{\circ}$ quartil) em comparação ao menor nível ( $1^{\circ}$ quartil).

\section{Peptídeo C e mortalidade cardiovascular}

A associação entre peptídeo C e mortalidade cardiovascular foi analisada em 11 estudos. Pontiroli et al. (27), em uma coorte de portadores de doença cardiovascular, demonstraram que os marcadores de resistência à insulina estavam associados à mortalidade. Bo et al. (28) acompanharam 931 pacientes diabéticos durante 14 anos e verificaram que níveis mais elevados do peptídeo $C$ reduziram o risco de complicações microvasculares, sem, contudo, produzir melhoria na sobrevida dos pacientes, não sendo observada associação entre os tercis do peptídeo $\mathrm{C}$ e a mortalidade geral ou por câncer, diabetes ou doença cardiovascular. Hirai et al. (29), na coorte multicêntrica de Wisconsin, acompanharam por 16 anos 1007 diabéticos e encontraram associação entre peptídeo $\mathrm{C}$ e mortalidade por doença cardíaca isquêmica somente nos homens (HR: 1,43; IC95\%: 1,13 a 1,83). Patel et al. (30), numa coorte retrospectiva com 51353 participantes, identificaram associação entre peptídeo C e mortalidade cardiovascular (HR: 1,72; IC95\%: 1,34 a 2,21). Nessa mesma direção, Marx et al. (31) encontraram maior risco para mortalidade cardiovascular (HR: 1,55; IC95\%: 1,07 a 2,24) nos dois tercis mais elevados de peptídeo C, após ajuste para vários fatores de confusão. Pikkemaat et al. (32), após ajuste para fatores de confusão, também observaram que pacientes no maior quartil de peptídeo $\mathrm{C}$ apresentaram mortalidade geral mais elevada (2,75 vezes) em comparação com aqueles no quartil inferior (IC95\%: 1,17 a 6,47).

Min e Min (33) encontraram associação entre os níveis séricos e mortalidade cardiovascular e geral em adultos não diabéticos, sugerindo um papel do peptídeo C como preditor de eventos fatais. Cardellini et al. (34), após identificarem um risco de 1,29 (IC95\%: 1,02 a 1,65) associado à mortalidade cardiovascular, sugeriram uma abordagem de reclassificação para considerar o peptídeo C como biomarcador para doenças cardiovasculares.

A partir dos estudos analisados, extraíram-se dados para compor a metanálise a respeito da associação entre peptídeo C e mortalidade cardiovascular a partir de seis estudos, com um total combinado de 15665 pacientes, obtendo-se uma razão de risco combinada para a mortalidade cardiovascular de 1,82 (IC95\%: 1,17 a 2,82) (figura 2).

A análise de subgrupos segundo a presença de comorbidade do diabetes e de potenciais portadores de doença cardíaca isquêmica mostrou risco relativo de 1,36 (IC95\%: 0,91 a 2,02) para o grupo com comorbidades e de 2,30 (IC95\%: 1,45 a 3,66) para o grupo sem comorbidades, conforme apresentado na figura 3.

\section{Peptídeo C e mortalidade geral}

Cinco estudos avaliaram a associação do peptídeo C com a mortalidade geral. Dois foram realizados em populações diabéticas e outros três em população livre de comorbidades cujos dados permitiram a inclusão nas análises desenvolvidas (figura 4). O efeito combinado foi de 2,92 (IC95\%: 2,02 a 4,21). A estimativa ajustada apresentou diminuição da medida de efeito (RR: 1,45; IC95\%: 1,35 a 1,54), porém manteve-se a significância da associação.

\section{DISCUSSÃO}

$\mathrm{Na}$ presente revisão sistemática, encontramos associação entre o peptídeo $\mathrm{C}$ e diferentes fatores de risco cardiovascular. 
FIGURA 2. Metanálise da mortalidade cardiovascular associada ao peptídeo C

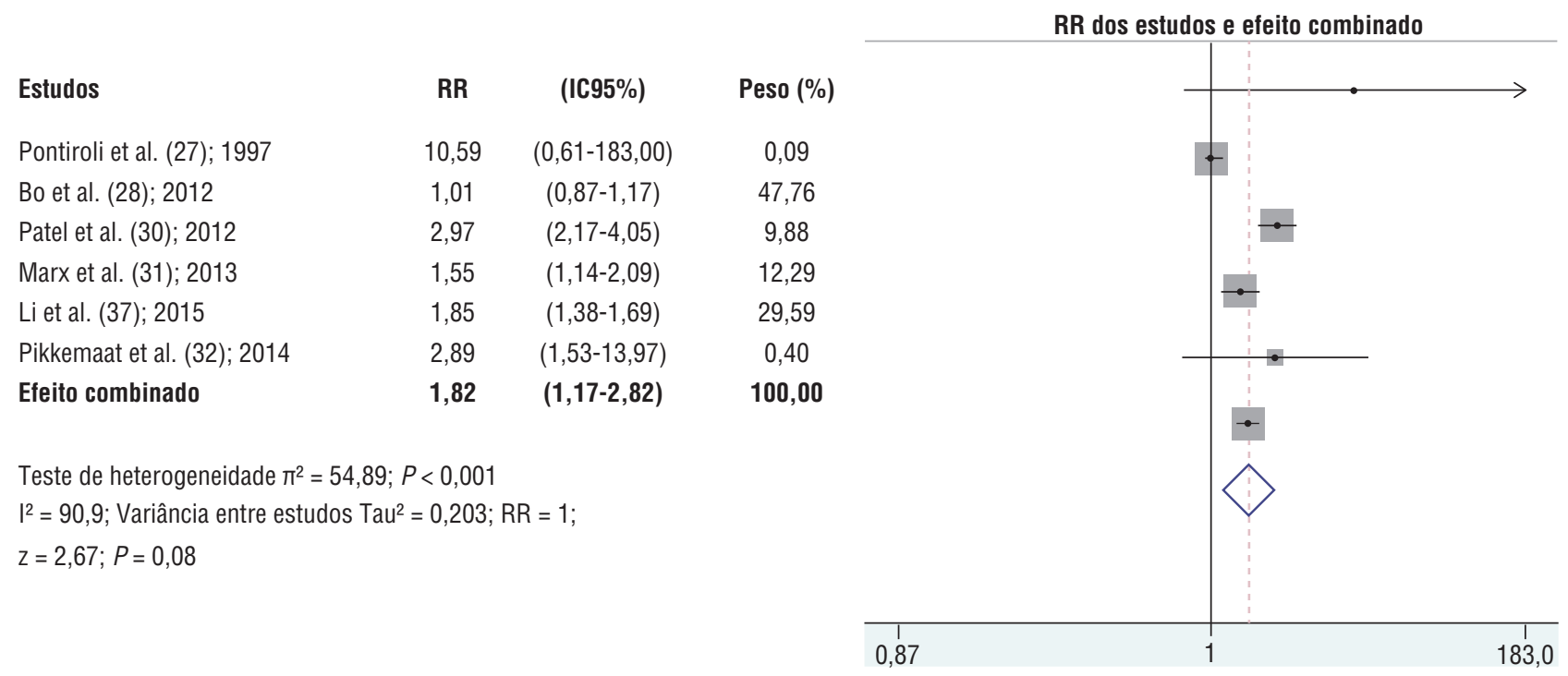

FIGURA 3. Metanálise da mortalidade cardiovascular segundo subgrupos de comorbidades

RR dos estudos e efeito combinado

\begin{tabular}{|c|c|c|c|}
\hline $\begin{array}{l}\text { Com comorbidades } \\
\text { Estudos }\end{array}$ & $\mathbf{R R}$ & (IC95\%) & Peso (\%) \\
\hline Pontiroli et al. (27); 1997 & 6,26 & $(0,35-112,70)$ & 1,82 \\
\hline Bo et al. (28); 2012 & 0,99 & $(0,89-1,12)$ & 43,12 \\
\hline Marx et al. (31); 2013 & 1,55 & $(1,15-2,10)$ & 35,57 \\
\hline Pikkemaat et al. (32); 2014 & 1,82 & $(0,92-3,58)$ & 19,49 \\
\hline Efeito combinado 1 & 1,36 & $(0,91-2,02)$ & 100,00 \\
\hline $\begin{array}{l}\text { Sem comorbidades } \\
\text { Estudos }\end{array}$ & RR & (IC95\%) & Peso (\%) \\
\hline Patel et al. (30); 2012 & 2,97 & $(2,97-4,05)$ & 46,37 \\
\hline Li et al. (37); 2015 & 1,85 & $(1,54-2,23)$ & 53,63 \\
\hline Efeito combinado 2 & 2,30 & $(1,45-3,66)$ & 100,00 \\
\hline Efeito combinado 3 & 1,75 & $(1,14-2,69)$ & \\
\hline
\end{tabular}

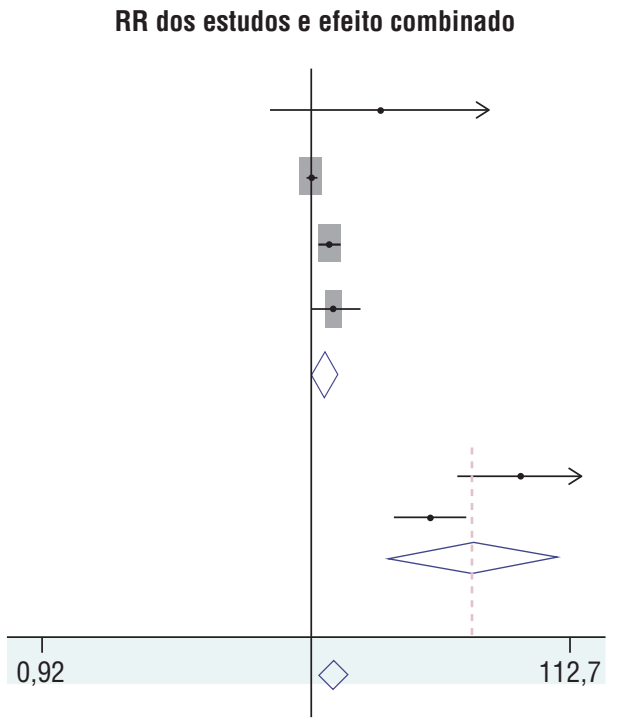

No entanto, o pequeno número de estudos e os diferentes critérios utilizados para definir a exposição impossibilitaram a realização de metanálise para os componentes de risco cardiovascular. Por outro lado, observamos associação positiva entre o peptídeo C e a mortalidade cardiovascular, com amostra de pouco mais de 15000 participantes investigados ao longo de um período médio de 10,9 anos de acompanhamento em estudos de base populacional. O peptídeo $\mathrm{C}$ aumentou o risco de mortalidade cardiovascular na análise de subgrupo em populações livres de comorbidade. A associação com risco de morte cardiovascular sugere que a resistência à insulina inferida pelos níveis de peptídeo $C$ favoreceria o desenvolvimento de eventos cardiovasculares $(30,31)$ e cardiovasculares fatais, como doença cardíaca isquêmica e infarto do miocárdio. Os mecanismos causais da associação entre peptídeo $\mathrm{C}$ e demais fatores de risco cardiovascular, como pressão arterial

sistólica, e componentes bioquímicos, como triglicerídeos, podem contribuir para o agravamento da disfunção endotelial, favorecendo os desfechos estudados $(36,37)$. Na presente revisão, o viés de múltipla publicação foi evitado pela exclusão das análises do estudo que empregava a mesma população do NHANES III (35).

$\mathrm{Na}$ metanálise foram empregadas as estimativas brutas e ajustadas para o estudo da mortalidade cardiovascular. Após os ajustes, a estimativa de efeito obtida ainda incluiu a unidade. Portanto, não se pode desconsiderar a hipótese de que a associação observada tenha sido decorrente do acaso. Cabe destacar a análise dos subgrupos, em que, na ausência de comorbidades, persistiu a associação entre peptídeo $\mathrm{C}$ e mortalidade cardiovascular e geral, demonstrando a inexistência de efeito de interação na análise combinada com essa condição (ausência de comorbidades). 
FIGURA 4. Metanálise da mortalidade geral associada ao peptídeo C

$\begin{array}{lccc}\text { Estudos } & \text { RR } & \text { (IC95\%) } & \text { Peso (\%) } \\ \text { Pontiroli et al. (27); } 1997 & 6,26 & (0,35-112,70) & 0,05 \\ \text { Bo et al. (28); 2012 } & 0,99 & (0,89-1,12) & 39,78 \\ \text { Patel et al. (30); 2012 } & 1,91 & (1,60-2,28) & 17,05 \\ \text { Min e Min (33); 2013 } & 1,41 & (1,26-1,57) & 41,94 \\ \text { Pikkemaat et al. (32); } 2014 & 1,82 & (0,93-3,58) & 1,17 \\ \text { Efeito combinado } & \mathbf{1 , 4 5} & & \mathbf{1 0 0 , 0 0}\end{array}$

RR dos estudos e efeito combinado

Teste de heterogeneidade $\pi^{2}=43,04 ; \mathrm{P}<0,001$

$I^{2}=90,7 \%$; Variância entre estudos $\operatorname{Tau}^{2}=0,083, \mathrm{RR}=1$;

$\mathrm{Z}=2,35 ; \mathrm{P}=0,019$

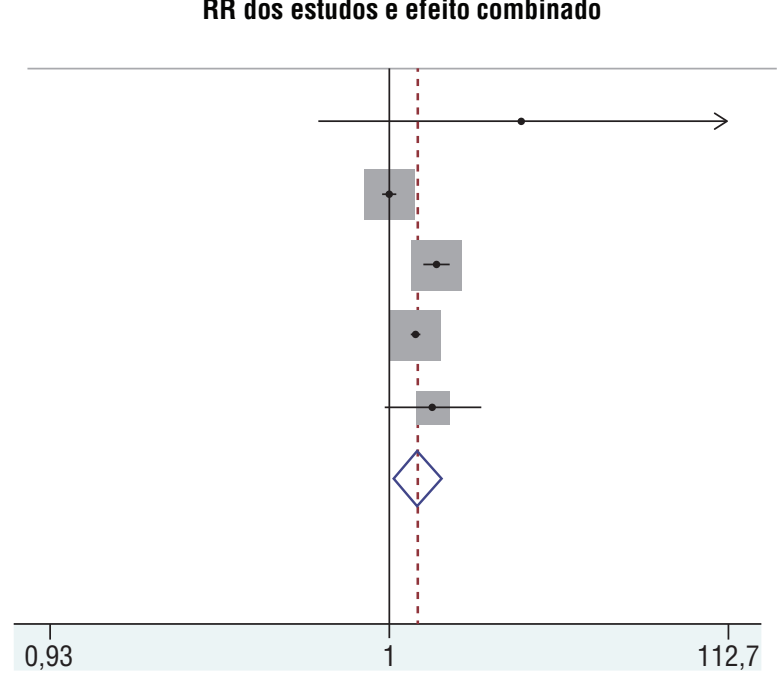

Limitações devem ser apontadas, no sentido de considerar os achados com cautela. É importante salientar a diversidade dos fatores de confusão nos estudos incluídos na metanálise e a não utilização de todos, particularmente os estudos que acompanharam pacientes com comorbidades - isso poderia ter favorecido a subestimação da medida de efeito nas medidas ajustadas, fortalecendo os presentes achados. Outro aspecto a ser comentado diz respeito ao pequeno número de estudos acerca do tema, o que aponta para a importância de novas investigações que contribuam com evidências válidas para a análise da associação entre peptídeo C e mortalidade cardiovascular e geral.

Em conclusão, os resultados desta metanálise, a primeira sobre o assunto, sugerem que o peptídeo C está associado à mortalidade geral para todos os indivíduos (RR: 1,39) e à mortalidade cardiovascular em pessoas sem comorbidades (RR: $2,30)$. Os achados contribuem para a compreensão da associação entre os níveis séricos de peptídeo C, considerados como substitutos da RI, e mortalidade cardiovascular e geral, permitindo recomendar a sua dosagem na prática clínica como proxy da condição de RI associada a mortalidade cardiovascular.

Contribuição dos autores. RLMA concebeu e desenhou a pesquisa, realizou a obtenção de dados e redigiu o artigo. GC realizou a obtenção de dados e redigiu o artigo. BLH desenhou

a pesquisa, avaliou as discordâncias no processo seletivo e redigiu o artigo. Todos os autores analisaram e interpretaram os dados, revisaram criticamente o conteúdo e aprovaram a versão final.

Agradecimentos. Os autores agradecem aos Drs. Simona Bo, Mirian Pikkemaat e Antonio Pontiroli, autores de artigos selecionados para compor a metanálise, pelo espírito colaborador e pela presteza no fornecimento dos dados que permitiram a realização das metanálise.

Conflito de interesse. Nada declarado pelos autores.

Financiamento. Programa de Doutorado Institucional da Coordenação de Aperfeiçoamento de Pessoal de Nível Superior (CAPES), através da cooperação técnica entre UFPel/ UFES, desenvolvido pelo Programa de Pós-Graduação em Epidemiologia da UFPel; e Wellcome Trust, por ocasião da criação da coorte de nascidos vivos da cidade de Pelotas, RS, Brasil.

Declaração. As opiniões expressas no manuscrito são de responsabilidade exclusiva dos autores e não refletem necessariamente a opinião ou política da RPSP/PAJPH ou da Organização Pan-Americana da Saúde (OPAS).

\section{REFERÊNCIAS}

1. Steiner DF, Cunningham D, Spigelman L, Aten B. Insulin biosynthesis: evidence for a precursor. Science. 1967;157(3789):697-700.

2. Horwitz DL, Rubenstein AH, Steiner DF. Proinsulin and C-peptide in diabetes. Med Clin North Am. 1978;62(4):723-33.

3. Clark PM. Assays for insulin, proinsulin(s) and C-peptide. Ann Clin Biochem. 1999;36(Pt5):541-64.

4. Kitabchi AE. Proinsulin and C-peptide: a review. Metabolism 1977;26(5):547-87.

5. Hills CE, Brunskill NJ. Intracellular signalling by C-peptide. Exp Diabetes Res. 2008;2008:635158.

6. Rodacki M, Zajdenverg L, Milech A, Oliveira JEP. Dosagem do peptídeo-C sérico ao acaso em adultos com diagnóstico clínico de diabetes mellitus tipo 1. Rev Assoc Med Bras. 2008;54(3):238-41.
7. Brandenburg D. History and diagnostic significance of C-Peptide. Exp Diabetes Res. 2008;2008:576862.

8. Hattersley, Jones AG, AT. The clinical utility of C-peptide measurement in the care of patients with diabetes. Diabet Med. 2013;30(7):803-17.

9. Sima AA, Zhang W, Sugimoto K, Henry D, Li Z, Wahren J, et al. C-peptide prevents and improves chronic Type I diabetic polyneuropathy in the BB/Wor rat. Diabetologia. 2001;44(7): 889-97.

10. Samnegard B, Jacobson SH, Jaremko G, Johansson BL, Ekberg K, Isaksson B, Eriksson L, Wahren J, Sjöquist M. C-peptide prevents glomerular hypertrophy and mesangial matrix expansion in diabetic rats. Nephrol Dial Transplant. 2005;20(3):532-8. 
11. Steffes MW, Sibley S, Jackson M, Thomas W. Beta-cell function and the development of diabetes-related complications in the diabetes control and complications trial. Diabetes Care. 2003;26(3):832-6.

12. Cai X, Han X, Zhang S, Luo Y, Chen Y, Ji L. Age at diagnosis and C-Peptide level are associated with diabetic retinopathy in Chinese. PLoS One. 2014;9(3):e91174.

13. Marx N, Walcher D, Raichle C, Aleksic M, Bach H, Grub M, et al. $\mathrm{C}$-peptide colocalizes with macrophages in early arteriosclerotic lesions of diabetic subjects and induces monocyte chemotaxis in vitro. Arterioscler Thromb Vasc Biol. 2004;24(3):540-5.

14. Vasic D, Walcher D. C-peptide: a new mediator of atherosclerosis in diabetes. Mediators Inflamm. 2012;2012:858692.

15. Haban P, Simoncic R, Zidekova E, Ozdin L. Role of fasting serum C-peptide as a predictor of cardiovascular risk associated with the metabolic X-syndrome. Med Sci Monit. 2002;8(3):CR175-9.

16. Ozougwu J, Obimba K, Belonwu C, Unakalamba C. The pathogenesis and pathophysiology of type 1 and type 2 diabetes mellitus. J Physiol Pathophysiol. 2013;4(4):46-57.

17. Lteif AA, Han K, Mather KJ. Obesity, insulin resistance, and the metabolic syndrome: determinants of endothelial dysfunction in whites and blacks. Circulation. 2005;112(1):32-8.

18. Von Eckardstein A, Fischer F, Schulte H, Tataru M, Kohler E, Assmann G. Association of serum apolipoprotein A-I (but not high-density lipoprotein cholesterol) with healed myocardial infarction in men independent of serum insulin and C-peptide. Am J Cardiol. 2001;88(7):723-6.

19. Cabrera de Leon A, Oliva Garcia JG, Marcelino Rodriguez I, Almeida Gonzalez D, Aleman Sánchez JJ, Brito Diaz B, et al. C-peptide as a risk factor of coronary artery disease in the general population. Diab Vasc Dis Res. 2015;12(3):199-207.

20. Stroup DF, Berlin JA, Morton SC, Olkin I, Williamson GD, Rennie D, et al. Meta-analysis of observational studies in epidemiology: a proposal for reporting. Meta-analysis Of Observational Studies in Epidemiology (MOOSE) group. JAMA. 2000;283(15):2008-12.

21. World Health Organization (WHO). Hearts: technical package for cardiovascular disease management in primary health care. Genebra: WHO; 2016. Disponível em: http://www.who.int/iris /handle/10665/252661 Acessado em 30 de setembro de 2018.

22. Wells GA, Shea B, O'Connell D, Peterson J, Welch V, Losos M, et al. The Newcastle-Ottawa Scale (NOS) for assessing the quality of nonrandomised studies in meta-analyses. Disponível em: http://www .ohri.ca/programs/clinical_epidemiology/oxford.asp Acessado em 30 de setembro de 2018.

23. Chen $\mathrm{CH}$, Tsai ST, Chou P. Correlation of fasting serum C-peptide and insulin with markers of metabolic syndrome- $X$ in a homogenous Chinese population with normal glucose tolerance. Int J Cardiol. 1999;68(2):179-86.

24. Chan WB, Tong PC, Chow CC, So WY, Ng MC, Ma RC, et al. The associations of body mass index, C-peptide and metabolic status in Chinese Type 2 diabetic patients. Diabet Med. 2004;21(4):349-53.

25. Abdullah A, Hasan H, Raigangar V, Bani-Issa W. C-Peptide versus insulin: relationships with risk biomarkers of cardiovascular disease in metabolic syndrome in young Arab females. Int J Endocrinol. 2012;2012:420792.
26. Li Y, Meng L, Zheng L. Association between serum C-Peptide as a risk factor for cardiovascular disease and high-density lipoprotein cholesterol levels in nondiabetic individuals. PLoS One. 2015;10(1):e112281.

27. Pontiroli AE, Pacchioni M, Camisasca R, Lattanzio R. Markers of insulin resistance are associated with cardiovascular morbidity and predict overall mortality in long-standing non-insulin-dependent diabetes mellitus. Acta Diabetol. 1998;35(1):52-6.

28. Bo S, Gentile L, Castiglione A, Prandi V, Canil S, Ghigo E, et al. $\mathrm{C}$-peptide and the risk for incident complications and mortality in type 2 diabetic patients: a retrospective cohort study after a 14-year follow-up. Eur J Endocrinol. 2012;167(2):173-80.

29. Hirai FE, Moss SE, Klein BE, Klein R. Relationship of glycemic control, exogenous insulin, and C-peptide levels to ischemic heart disease mortality over a 16-year period in people with older-onset diabetes: the Wisconsin Epidemiologic Study of Diabetic Retinopathy (WESDR). Diabetes Care. 2008;31(3):493-7.

30. Patel N, Taveira TH, Choudhary G, Whitlatch H, Wu WC. Fasting serum C-peptide levels predict cardiovascular and overall death in nondiabetic adults. J Am Heart Assoc. 2012;1(6): e003152.

31. Marx N, Silbernagel G, Brandenburg V, Burgmaier M, Kleber ME, Grammer TB, et al. C-peptide levels are associated with mortality and cardiovascular mortality in patients undergoing angiography: the LURIC study. Diabetes Care. 2013;36(3):708-14.

32. Pikkemaat M, Melander O, Mölstad S, Garberg G, Boström KB. C-peptide concentration, mortality and vascular complications in people with type 2 diabetes. The Skaraborg Diabetes Register. Diabet Med. 2015;32(1):85-9.

33. Min J, Min K. Serum C-Peptide levels and risk of death among adults without diabetes mellitus. CMAJ. 2013;185(9): E402-8.

34. Cardellini M, Farcomeni A, Ballanti M, Morelli M, Davato F, Cardolini I, et al. C-peptide: a predictor of cardiovascular mortality in subjects with established atherosclerotic disease. Diab Vasc Dis Res. 2017;14(5):395-9.

35. Panero F, Novelli G, Zucco C, Fornengo P, Perotto M, Segre O, et al. Fasting plasma C-Peptide and micro-and macrovascular complications in a large clinic-based cohort of type 1 diabetic patients. Diabetes Care. 2009;32(2):301-5.

36. Donatelli M, Scarpinato A, Bucalo ML, Russo V, Iraci T, Vassallo G. Stepwise increase in plasma insulin and C-peptide concentrations in obese, in obese hypertensive, and in obese hypertensive diabetic subjects. Diabetes Res. 1991;17(3):125-9.

37. Li Y, Meng L, Sato Y. Associations of serum C-peptide level with body fat distribution and ever stroke in nondiabetic subjects. J Stroke Cerebrovasc Dis. 2014;23(3):e163-9.

Manuscrito recebido em 23 de março de 2018. Aceito em versão revisada em 25 de outubro de 2018 . 


\section{C-peptide and cardiovascular mortality: systematic review and meta-analysis}

ABSTRACT Objective. To analyze the available evidence regarding the association between C-peptide and cardiovascular and overall mortality.

Method. A systematic review of MEDLINE and EMBASE was performed. Articles published in English, Portuguese, or Spanish, reporting observational studies investigating the association between C-peptide and cardiovascular or overall mortality were included. The association between C-peptide and cardiometabolic risk factors, hemodynamic factors, and anthropometric measures was also investigated. The methodological quality of studies was assessed using the Newcastle-Ottawa Scale.

Results. The literature search revealed 107 articles on the topic of interest. Following the screening step, 18 articles presenting data on the association between C-peptide and cardiovascular risk were included. Five studies provided data on the relationship between C-peptide and cardiovascular or overall mortality. C-peptide was positively associated with body mass index in Chinese individuals, and inversely associated with HDL cholesterol in population samples from Asia, Middle East, and the United Stated. Nevertheless, meta-analysis of cardiovascular risk components was not possible. In the meantime, C-peptide was associated with cardiovascular mortality (RR $=1.62 ; 95 \% \mathrm{Cl}: 0.99-2.66)$ and overall mortality $(\mathrm{RR}=1.39 ; 95 \% \mathrm{Cl}: 1.04-1.84)$.

Conclusion. The present systematic review and meta-analysis showed that serum levels of C-peptide were positively associated with overall mortality in all individuals and with cardiovascular mortality in individuals without comorbidities. Based on these results, it is possible to recommend the use of C-peptide in clinical practice as a proxy of insulin resistance associated with cardiovascular mortality.

Keywords $\quad$ C-peptide; mortality; cardiovascular diseases; meta-analysis.

\section{Péptido C y mortalidad cardiovascular: revisión sistemática y metanálisis}

RESUMEN Objetivo. Analizar las evidencias sobre la asociación entre el péptido C y la mortalidad cardiovascular y general disponibles en la bibliografía.

Métodos. Se realizó una revisión sistemática en las bases de datos MEDLINE y EMBASE. Se incluyeron artículos publicados en inglés, portugues o español con estudios observacionales que investigaron la asociación entre el péptido C y la mortalidad cardiovascular y general. Se buscó también evaluar la asociación entre el péptido $\mathrm{C}$ y factores de riesgo cardiometabólicos, factores hemodinámicos y medidas antropométricas. Se evaluó la calidad metodológica de los estudios según los criterios de la escala de Newcastle-Ottawa.

Resultados. Se identificaron 107 estudios. Al final del proceso de clasificación se incluyeron 18 artículos que presentaban datos sobre la asociación entre el péptido C y el riesgo cardiovascular. Cinco estudios proporcionaron datos sobre la relación entre el péptido C y la mortalidad cardiovascular y general. El péptido C presentó una asociación positiva con el índice de masa corporal en población china, y una asociación inversa con el colesterol HDL en muestras poblacionales de Asia, Oriente Medio y Estados Unidos. Sin embargo, no fue posible realizar un metanálisis para los componentes de riesgo cardiovascular. Por otro lado, el péptido C se asoció con la mortalidad cardiovascular $(R R=1,62, I C 95 \%: 0,99$ a 2,66) y general $(R R=1,39, I C 95 \%$ : 1,04 a 1,84).

Conclusión. En la revisión sistemática y el metanálisis realizados los niveles séricos de péptido C se asociaron positivamente con la mortalidad general en todos los individuos y con la mortalidad cardiovascular en personas sin comorbilidades. Con base en estos resultados, es posible recomendar el empleo del péptido C en la práctica clínica como proxy de la resistencia a la insulina asociada a la mortalidad cardiovascular.

Palabras clave Péptido C; mortalidad; enfermedades cardiovasculares; metaanálisis. 